\title{
Editorial
}

\section{Introduction to the special issue: Officials in sports}

The history of sport referees, judges, and officials, began with the first Olympic Games in the Antiquity. The Hellanodikai, the judges of the Greeks, had many roles including; selecting the athletes, supervising their training, coordinating the drawing lots, refereeing the competitions and judging and deciding on sanctions if it was required (Bagnall, Brodersen, Champion, Erskine, \& Huebner, 2013). Nowadays, thousands of sports officials (referees, judges, and umpires) work every week in the majority of competitive sports. In recent years, the amount of research within the area has increased, and the empirical evidence accumulated reflects the diversity of refereeing as well as its complexity (Dosseville \& Laborde, 2011).

Pioneer work in the seventies focused on basketball referees' personality (Alker, Straub, \& Leary, 1973; Fratzke, 1975). From this, sport officials came to forefront of attention for researchers. Around 60 scientific papers were published between 1980 and 1990 with a focus on personality (Ittenbach \& Eller, 1989), stress (Kaissidis \& Anshel, 1993; Taylor, Daniel, Leith, \& Burke, 1990), the decision-making process and biases that may influence it (Larsen \& Rainey, 1991; Ste-Marie \& Lee, 1991). During this time two books ${ }^{1}$ were published, summarizing guidance for physical and psychological training of referees, and analyzing the communication with the other sport actors (Grunska, 1999; Weinberg \& Richardson 1990). From 2000 on, the literature started to grow and focused on aspects linked to judgment and decision-making as well as on physical and physiological dimensions. Expertise (MacMahon \& Plessner, 2008; Ollis, MacPherson, \& Collins, 2006; Ste-Marie, 2003), previous experience (Catteeuw, Helsen, Gilis, \& Wagemans, 2009; MacMahon, Helsen, Starkes, Weston, 2007; Pizzera \& Raab, 2012), stress and coping strategies (Louvet, Gaudreau, Menaut, Genty, \& Deneuve, 2009; Voight, 2009) and visual perception of referees (Helsen, Gilis, \& Weston, 2006; Oudejans et al., 2000) were thus investigated and debated amongst the scientific community. This work in psychology allowed for the investigation of influencing internal and external factors including: stereotypes (Souchon, 2011; Souchon et al., 2009), reputation (Findlay \&

\footnotetext{
1 A first book was published in 1950 by Bunn called The Art of Officiating.
}

Ste-Marie, 2004; Jones, Paull, \& Erskine, 2002), visual and motor experience (Pizzera \& Laborde, 2011; Pizzera \& Raab, 2012), audience (Goumas, 2012; Nevill, Balmer, \& Williams, 1999; Unkelbach \& Memmert, 2012) and context (Brand, Schmidt, \& Schneeloch, 2006; Dosseville, Laborde, \& Raab, 2011). Finally, referees' motivation (Gray \& Wilson, 2008; Philippe, Vallerand, Andrianarisoa, \& Brunel, 2009) and communication, in particular with the other actors of sport competitions (Cunningham, Simmons, Mascarenhas, \& Redhead 2014; Dosseville, Laborde, \& Bernier, 2014; Mellick, Fleming, \& Davies, 2007; Simmons \& Cunningham, 2013) were also recently the focus for researchers in this field.

Regarding the physiological aspects of refereeing, researchers investigated the characteristics of referees' physical activity, and the physiological demands during sporting competition (Krustrup \& Bangsbo, 2001; Martin, Smith, Tolfrey, \& Jones, 2001). The investigation of physical workload (Castagna, Abt, \& D'Ottavio, 2007; Krustrup, et al., 2009; Leicht, 2008; Weston, Castagna, Impellizzeri, Rampinini, \& Breivik, 2010; Weston, Drust, \& Gregson, 2011) and the evaluation of training tools (Castagna, Abt, \& d'Ottavio, 2005; Weston, Helsen, MacMahon, \& Kirkendall, 2004) have focused on team sports such as soccer, rugby and basketball.

Other works also investigated the psychological and physiological aspects linked to referees' decision-making (Dosseville, Laborde, Traclet, \& Edoh, 2011; Helsen \& Bultynck, 2004; Mascarenhas, Button, O'Hare, \& Dicks, 2009). Moreover, some literature reviews on refereeing and chapters that summarize the knowledge on refereeing were published during the last decade (Bar-Eli, Plessner, \& Raab, 2010; Dosseville \& Garncarzyk, 2007; Dosseville, Laborde, \& Garncarzyk, 2014; Guillén \& Feltz, 2011; Mascarenhas, Collins, \& Mortimer, 2005; Plessner \& Haar, 2006). The current reviews underline the importance of the findings within refereeing and officiating research and demonstrate that it is a multidisciplinary area which still has significant research gaps that need addressing.

This special issue of Movement \& Sport Sciences therefore aims to contribute to the growing literature on sport officials, to stimulate and encourage researchers' interest for this area of research within the field of sport 
sciences. Therefore a diverse range of scientific theoretical approaches and methodologies are presented which examine officials from different sports, in papers written in both French and English. In total, we received 25 abstracts for the special issue, 22 full papers were then submitted, from which 10 (six in English and four in French) passed the selection and reviewing process, and were included in this special issue.

The paper of Ludovic Ténèze, Hélène Joncheray and Thierry Arnal starts this special issue with a historical perspective regarding the institutionalization of soccer referees. Based on the archives from the most ancient soccer clubs and from the Board, the authors evaluate the evolution of the role and power of the referee in relationship to the history of the laws of the game. The evolution of soccer referees responsibility allows for an understanding of the complexity of refereeing until the development of specific modern technologies.

One of the biggest questions nowadays amongst sport associations is related to career length and high dropout rates among officials in a large range of sports. In order to understand and explain what drives referees to start and to dropout, the motivation linked to refereeing is the focus of three papers. Firstly, Bjørn Tore Johansen examines this issue primarily with a quantitative approach and examines the reasons why elite and non-elite referees began to referee. He found three main reasons for this: passion, social support and health. Subsequently, he then discusses the differences according to referees' expertise level. Secondly, David Hancock, Donald J. Dawson and Denis Auger investigated the different motivations to start, continue and to stop refereeing in 18 sports. Using a quantitative approach, they underline the importance of several key variables such as intrinsic motivation, stress and lack of respect. Finally, based on a literature review and interviews, Tom Parsons and Alan Bairner focused on the engagement of British soccer referees: ambition, satisfaction, and social support are discussed as essential elements of engaging in refereeing.

Expertise and stress are then considered. Alexandra Pizzera investigated the expertise of sport officials through the theoretical lens of embodied cognition, which is rarely considered in regard to referees. Embodied cognition assumes that the comprehension of expertise should also undergo the investigation of visual expertise, motor expertise, and refereeing expertise. Moreover, Benoît Louvet, Mickaël Campo and Amaël André looked at the dispositional and situational factors influencing coping strategies in soccer referees. Using structural equation modeling, the authors show that the perception of game stakes and the intensity of anxiety predict the coping strategies used, while perfectionism remains the only single variable directly influencing these strategies.

Communication is at the heart of questions faced by sport associations, but remains largely unexplored by sport sciences researchers. Therefore, three papers focus on this topic: in the first study, Ian Cunningham, Peter Simmons, Duncan Mascarenhas and Steve Redhead use the sociology of social interactions to explore the interactions between referees of team sports and the players. They observed that players often try to influence referees with several attitudes, thus improving the understanding of communication and interactions in the field. Simon Boyer, Géraldine Rix-Lièvre and Michel Recopé examined the coordination of the contribution between the different members of referees' teams in soccer. Using self-confrontation interviews, the authors offer a first insight regarding the contribution of each official to the refereeing of an elite-level soccer game. Finally, François Borel-Hänni and Fabien Wille investigated communication within archives and interviews. More specifically, they asked soccer referees about their representation in the media, and highlight that television media is mainly perceived negatively by soccer referees.

To conclude this special issue, a review by Matthew Weston investigates the physical, perceptual and cognitive performance of soccer referees, which considers those aspects as being intrinsically linked. The author considered the quantification of physical workload, the decisionmaking process, the training plan and different training tools. He then raises the question of fatigue, explaining the difficulty to examine its influence on judgment and decision-making in ecological conditions, and also contemplates the methods about decision-making training.

This special issue aims to showcase the diversity and complementary aspects of the different approaches to the research area. Overall, decisions appear to have a central role for sport officials, being considered either on a longterm or on a short-term basis. Long-term decisions are related to career choices, dropout and transitions whereas short-term decisions are related to decisions made on the field. Short terms decisions are dynamic and made in an ecological environment, where time, space, and uncertainty constraints are multiple and potentially interact together, which makes cue processing quite difficult. We aspire that this special issue will stimulate new research questions in the domain of sport science and trigger interest in researchers, students, and practitioners. Furthermore, we hope that it will foster collaborations between the different research teams contributing to this special issue and those who would like to contribute to this field in the future. We would finally like to thank the Editorin-Chief of Movement 83 Sport Sciences for giving us the opportunity to serve as Guest Editors for this special issue on sport officials.

Acknowledgements. We would like to thank Ms. Emma Mosley, University of Bournemouth (UK) for her helpful comments on a first draft version of this introduction.

Fabrice Dosseville ${ }^{1}$ and Sylvain Laborde $\mathrm{e}^{1,2}$ ${ }^{1}$ Université de Caen Basse-Normandie

${ }^{2}$ German Sport University Cologne 


\section{Bibliography}

Alker, H.A., Straub, W.F., \& Leary, J. (1973). Achieving consistency: A Study of basketball officiating. Journal of Vocational Behavior, 3 (3), 335-343.

Bagnall, R.S., Brodersen, K., Champion, C.B., Erskine, A., \& Huebner, S.R. (2013). The Encyclopedia of Ancient History. Malden, MA: Wiley-Blackwell.

Bar-Eli, M., Plessner, H., \& Raab, M. (2010). Referees. In M. Bar-Eli, H. Plessner, \& M. Raab (Eds.), Judgment, decision-making and success in sport (pp. 123-144). Hoboken, NJ: John Wiley \& Sons, Ltd.

Brand, R., Schmidt, G., \& Schneeloch, Y. (2006). Sequential effects in elite basketball referees' foul decisions: An experimental study on the concept of game management. Journal of Sport and Exercise Psychology, 28 (1), 93-99.

Castagna, C., Abt, G., \& D'Ottavio, S. (2005). CompetitiveLevel Differences in Yo-Yo Intermittent Recovery and Twelve Minute Run Test Performance in Soccer Referees. Journal of Strength and Conditioning Research, 19 (4), 805-809.

Castagna, C., Abt, G., \& D'Ottavio, S. (2007). Physiological aspects of soccer refereeing performance and training. Sports Medicine, 37 (7), 625-647.

Catteeuw, P., Helsen, W., Gilis, B., \& Wagemans, J. (2009). Decision-making skills, role specificity, and deliberate practice in association football refereeing. Journal of Sports Sciences, 27 (11), 1125-1136.

Cunningham, I., Simmons, P., Mascarenhas, D. \& Redhead, S. (2014). Concepts of communication and player management in the development of sport officials. International Journal of Sport Communication, 7 (2), 166-187.

Dosseville, F., \& Garncarzyk, C. (2007). L'arbitrage des pratiques sportives : Jugement et décision. Bulletin de Psychologie, 60 (3), 225-237.

Dosseville, F., \& Laborde, S. (2011). Les facettes de l'arbitrage : Recherches et problématiques actuelles. Paris: Publibook, Collection Université: Sport \& Santé.

Dosseville, F., Laborde, S., \& Bernier, M. (2014). Athletes' expectations with regard to officiating competence. European Journal of Sport Science, 14 (S1), S448-S455.

Dosseville, F., Laborde, S., \& Garncarzyk, C. (2014). Current research in Sports officiating and decision-making. In $\mathrm{C}$. Mohiyeddini (Ed.), Contemporary Topics and Trends in the Psychology of Sports (pp. 13-38). New York: Nova Publishers.

Dosseville, F., Laborde, S., \& Raab, M. (2011). Contextual and personal motor experience effects in judo referees' decisions. The Sport Psychologist, 25 (1), 67-81.

Dosseville, F., Laborde, S., Traclet, A., \& Edoh, K.P. (2011). Décisions arbitrales en football et performances physiques en situation écologique : Indicateurs comportementaux et physiologiques. STAPS, Revue Internationale des Sciences du Sport 8 de l'Education Physique, 93 (3), 51-60.

Findlay, L.C., \& Ste-Marie, D.M. (2004). A reputation bias in figure skating judging. Journal of Sport and Exercise Psychology, 26 (1), 154-166.
Fratzke, M.R. (1975). Personality and biographical traits of superior and average college basketball officials. The Research Quarterly, 46, 484-488.

Goumas, C. (2012). Home advantage and referee bias in European football. European Journal of Sport Science, 14 (sup1), S243-S249.

Gray, C.E., \& Wilson, P.M. (2008). The relationship between organizational commitment, perceived relatedness, and intentions to continue in Canadian track and field officials. Journal of Sport Behavior, 31, 44-63.

Grunska, J. (1999). Successful sports officiating. Champaign, IL: Human Kinetics.

Guillén, F., \& Feltz, D.L. (2011). A conceptual model of referee efficacy. Frontiers in Psychology, 2 (25), 1-5.

Helsen, W., \& Bultynck, J.-B. (2004). Physical and perceptual-cognitive demands of top-class refereeing in association football. Journal of Sports Sciences, 22 (2), 179-189.

Ittenbach, R.F., \& Eller, B.F. (1989). The Official Personality. Journal of Sport and Exercise Psychology, 11 (2), 119-119.

Jones, M.V., Paull, G.C., \& Erskine, J. (2002). The impact of a team's aggressive reputation on the decisions of association football referees. Journal of Sports Sciences, 20 (12), 991-1000.

Kaissidis, A., \& Anshel, M.H. (1993). Sources and intensity of acute stress in adolescent and adult Australian basketball referees: a preliminary study. Australian Journal of Science and Medicine in Sport, 25 (3), 97-103.

Krustrup, P., \& Bangsbo, J. (2001). Physiological demands of top-class soccer refereeing in relation to physical capacity: effect of intense intermittent exercise training. Journal of Sports Sciences, 19 (11), 881-891.

Krustrup, P., Helsen, W., Randers, M.B., Christensen, J.F., MacDonald, C., Rebelo, A.N., \& Bangsbo, J. (2009). Activity profile and physical demands of football referees and assistant referees in international games. Journal of Sports Sciences, 27 (11), 1167-1176.

Larsen, J.D., \& Rainey, D.W. (1991). Judgment Bias in Baseball Umpires' First Base Calls: A Computer Simulation. Journal of Sport and Exercise Psychology, 13 (1), 75-79.

Leicht, A.S. (2008). Physiological demands of basketball refereeing during international competition. Journal of Science and Medicine in Sport, 11 (3), 357-360.

Louvet, B., Gaudreau, P., Menaut, A., Genty, J., \& Deneuve, P. (2009). Revisiting the changing and stable properties of coping utilization using latent class growth analysis: A longitudinal investigation with soccer referees. Psychology of Sport and Exercise, 10, 124-135.

MacMahon, C., \& Plessner, H. (2008). The sport officials in research and practice. In D. Farrow, J. Baker \& C. MacMahon (Eds.), Developing sport expertise. Researchers and coaches put theory into practice (pp. 172-192). Abingdon, Oxon: Routledge. 
Martin, J., Smith, N.C., Tolfrey, K., \& Jones, A.M. (2001). Activity analysis of English premiership rugby football union refereeing. Ergonomics, 44 (12), 1069-1075.

Mascarenhas, D.R.D., Button, C., O'Hare, D., \& Dicks, M. (2009). Physical performance and decision making in association football referees: A naturalistic study. The Open Sports Sciences Journal, 2, 1-9.

Mascarenhas, D.R.D., Collins, D., \& Mortimer, P.W. (2005). Elite Refereeing Performance: Developing a model for sport science support. The Sport Psychologist, 19 (4), $364-379$.

Mellick, M.C., Fleming, S., \& Davies, G. (2007). An interpretive analysis of interpersonal communication: A case study from elite rugby union match officiating. International Journal of Performance Analysis in Sport, 7 (2), 92-105.

Nevill, A.M., Balmer, N.J., \& Williams, A.M. (1999). Crowd influence on decisions in association football. The Lancet, 353 (9162), 1416-1416.

Ollis, S., MacPherson, A., \& Collins, D. (2006). Expertise and talent development in rugby refereeing: an ethnographic enquiry. Journal of Sports Sciences, 24 (3), 309-322.

Oudejans, R.R.D., Verheijen, R., Bakker, F.C., Gerrits, J.C., Steinbrückner, M., \& Beek, P.J. (2000). Errors in judging 'offside' in football. Nature, 404, 33.

Philippe, F.L., Vallerand, R.J., Andrianarisoa, J., \& Brunel, P. (2009). Passion in referees: Examining their affective and cognitive experiences in sport situations. Journal of Sport and Exercise Psychology, 31 (1), 77-96.

Pizzera, A., \& Laborde, S. (2011). Comment devient-on expert en arbitrage ? In F. Dosseville \& S. Laborde (Eds.), Les facettes de l'arbitrage: Recherches et problématiques actuelles (pp. 134-146). Paris : Publibook, Collection Université.

Pizzera, A., \& Raab, M. (2012). Perceptual judgments of sports officials are influenced by their motor and visual experience. Journal of Applied Sport Psychology, 24, 59-772.

Plessner, H., \& Haar, T. (2006). Sports performance judgments from a social cognitive perspective. Psychology of Sport and Exercise, 7, 555-575.

Simmons, P., \& Cunningham, I. (2013). Communication and sports officials. In P.M. Pedersen, Routledge handbook of sport communication (pp. 461-470). London : Routledge.
Souchon, N., Cabagno, G., Rascle, O., Traclet, A., Dosseville, F., \& Maio, G.R. (2009). Referees' decision making about transgressions: The influence of player at the highest national level. Psychology of Women Quarterly, 33 (4), $445-452$.

Souchon, N. (2011). Stéréotypes, attitudes, préjugés et décisions arbitrales. In F. Dosseville \& S. Laborde (Eds.), Les facettes de l'arbitrage: Recherches et problématiques actuelles (pp. 113-113). Paris: Publibook, Collection Université.

Ste-Marie, D.M. (2003). Expertise in sport judges and referees. Circumventing information-processing limitations. In J.L. Starkes \& K.A. Ericsson (Eds.), Expert performance in sports: Advances in research on sport expertise (pp. 169-190). Champaign, IL: Human Kinetics.

Ste-Marie, D.M., \& Lee, T.D. (1991). Prior processing effects on gymnastic judging. Journal of Experimental Psychology: Learning, Memory \& Cognition, 17 (1), 126-136.

Taylor, A.H., Daniel, J.V., Leith, L., \& Burke, R.J. (1990). Perceived stress, psychological burnout and paths to turnover intentions among sport officials. Journal of Applied Sport Psychology, 2 (1), 84-97.

Unkelbach, C., \& Memmert, D. (2010). Crowd noise as a cue in referee decisions contributes to the home advantage. Journal of Sport and Exercise Psychology, 32 (4), 483-498.

Voight, M. (2009). Sources of stress and coping strategies of US soccer officials. Stress and Health, 25 (1), 91-101.

Weinberg, R.S., \& Richardson, P.A. (1990). Psychology of officiating. Champaign, ILL: Leisure Press.

Weston, M., Castagna, C., Impellizzeri, F.M., Rampinini, E., \& Breivik, S. (2010). Ageing and physical match performance in English Premier League soccer referees. Journal of Science and Medicine in Sport, 13 (1), 96-100.

Weston, M., Drust, B., \& Gregson, W. (2011). Intensities of exercise during match-play in FA Premier League referees and players. Journal of Sports Sciences, 29 (5), 527-532.

Weston, M., Helsen, W., MacMahon, C., \& Kirkendall, D. (2004). The impact of specific high-intensity training sessions on football referees' fitness levels. American Journal of Sports Medicine, 32 (1 Suppl.), 54S-61S. 


\section{Éditorial}

\section{Introduction au numéro spécial : les officiels des pratiques sportives}

L'histoire des arbitres, juges et officiels des pratiques sportives débute avec les jeux antiques. Les Hellanodikai, littéralement juges des Grecs, sélectionnaient les athlètes et supervisaient leur entraînement, effectuaient les tirages au sort, dirigeaient les rencontres, jugeaient et choisissaient les sanctions le cas échéant (Bagnall, Brodersen, Champion, Erskine, \& Huebner, 2013).. Aujourd'hui, des milliers d'arbitres et de juges officient chaque semaine dans la plupart des sports à visée compétitive. Depuis quelques années, les regards scientifiques se multiplient et soulignent la multitude des facettes de l'arbitrage des pratiques sportives ainsi que la complexité de cette activité essentielle (Dosseville \& Laborde, 2011)

Après de premiers travaux au milieu des années 1970 centrés sur la personnalité des arbitres de basketball (Alker, Straub, \& Leary, 1973; Fratzke, 1975), l'officiel des pratiques sportives (referee, umpire, judge) devient l'objet de plus en plus de travaux scientifiques. Une soixante d'articles va ainsi être publiée dans les années 1980 et 1990 se focalisant sur la personnalité (Ittenbach \& Eller, 1989), le stress (Kaissidis \& Anshel, 1993; Taylor, Daniel, Leith, \& Burke, 1990), le processus décisionnel et ses différents biais (Larsen \& Rainey, 1991; Ste-Marie \& Lee, 1991). C'est à cette même époque que seront publiés deux ouvrages ${ }^{1}$ regroupant à la fois des conseils sur la préparation physique et psychologique de l'arbitre et la communication avec les autres acteurs de la rencontre sportive (Grunska 1999; Weinberg \& Richardson 1990). À partir des années 2000, la littérature s'étend et se focalise sur les processus de jugement et de prise de décision ainsi que sur les aspects physiques et physiologiques.

L'expertise (MacMahon \& Plessner, 2008; Ollis, MacPherson, \& Collins, 2006; Ste-Marie, 2003), les expériences (Catteeuw, Helsen, Gilis, \& Wagemans, 2009 ; MacMahon, Helsen, Starkes, Weston, 2007; Pizzera \& Raab, 2012), le stress et les stratégies de coping (Louvet, Gaudreau, Menaut, Genty, \& Deneuve, 2009; Voight, 2009) et la perception visuelle des arbitres (Helsen, Gilis,

\footnotetext{
${ }^{1}$ Un premier ouvrage avait été publié en 1950 par Bunn intitulé The Art of Officiating.
}

\& Weston, 2006; Oudejans, et al., 2000) font ainsi l'objet de nombreux travaux et débats dans la communauté scientifique. Ces travaux en psychologie permettent ainsi d'examiner l'influence de facteurs internes et externes : les stéréotypes (Souchon, 2011; Souchon, et al., 2009), la réputation (Findlay \& Ste-Marie, 2004; Jones, Paull, \& Erskine, 2002), les expériences motrices et visuelles (Pizzera \& Laborde, 2011; Pizzera \& Raab, 2012), le public (Goumas, 2012; Nevill, Balmer, \& Williams, 1999; Unkelbach \& Memmert, 2012) ou le contexte (Brand, Schmidt, \& Schneeloch, 2006; Dosseville, Laborde, \& Raab, 2011). Enfin, les motivations des arbitres (Gray \& Wilson, 2008; Philippe, Vallerand, Andrianarisoa, \& Brunel, 2009) ainsi que la communication, notamment avec les autres acteurs des rencontres sportives (Cunningham, Simmons, Mascarenhas, \& Redhead 2014; Dosseville, Laborde, \& Bernier, 2014; Mellick, Fleming, \& Davies, 2007; Simmons \& Cunningham, 2013) ont également élargi le champ d'investigation des chercheurs ces dernières années.

Du côté de la physiologie de l'effort, les chercheurs analysent l'activité physique des arbitres et les demandes physiologiques lors des rencontres (Krustrup \& Bangsbo, 2001; Martin, Smith, Tolfrey, \& Jones, 2001). L'étude de la charge de travail et de l'intensité physique (Castagna, Abt, \& D'Ottavio, 2007; Krustrup, et al., 2009; Leicht, 2008; Weston, Castagna, Impellizzeri, Rampinini, \& Breivik, 2010; Weston, Drust, \& Gregson, 2011) ainsi que l'évaluation des outils d'entraînement (Castagna, Abt, \& d'Ottavio, 2005; Weston, Helsen, MacMahon, \& Kirkendall, 2004) se sont ainsi focalisées sur les sports collectifs tels que le football, le rugby et le basketball. D'autres travaux ont également lié les aspects physiques et physiologiques à la prise de décision arbitrale (Dosseville, Laborde, Traclet, \& Edoh, 2011; Helsen \& Bultynck, 2004; Mascarenhas, Button, O'Hare, \& Dicks, 2009).

Finalement, quelques revues de littérature sur l'arbitrage et le jugement ou chapitres d'ouvrages synthétisant nos connaissances sur les arbitres des pratiques sportives ont été publiés récemment (Bar-Eli, 
Plessner, \& Raab, 2010; Dosseville \& Garncarzyk, 2007; Dosseville, Laborde, \& Garncarzyk, 2014; Guillén \& Feltz, 2011; Mascarenhas, Collins, \& Mortimer, 2005; Plessner \& Haar, 2006). Toutefois, si ces états de l'art soulignent l'importance de travaux en sciences du sport et du mouvement, ils montrent également que les perspectives restent très larges et que l'arbitrage et les officiels sont devenus des objets de recherche pluridisciplinaire.

Ce numéro spécial de Movement \& Sport Sciences a donc pour objectif de contribuer à la littérature croissante qui aborde les officiels des pratiques sportives, de stimuler et favoriser l'intérêt des chercheurs pour cet acteur et son activités dans le champ des sciences du sport et du mouvement. Il convoque ainsi diverses approches, ancrages et méthodes scientifiques, des disciplines sportives différentes, en langue française ou anglaise. Vingt cinq résumés puis 22 articles complets ont été soumis. Finalement, 10 articles (6 en anglais et 4 en français) ont passé l'ensemble des étapes d'expertises et ont permis ce numéro spécial.

La contribution de Ludovic Ténèze, Hélène Joncheray et Thierry Arnal ouvre ce numéro spécial avec une approche historique. Elle est celle d'historiens s'intéressant au processus d'institutionnalisation de l'arbitre de football. Sur la base d'archives des premiers clubs de football ainsi que sur celles du Board, ces auteurs abordent l'évolution du rôle et du pouvoir de l'arbitre en relation avec l'histoire des lois du jeu. L'évolution des responsabilités des arbitres de football permettent alors d'appréhender la complexité de l'arbitrage jusqu'aux récents apports de la technologie.

L'une des grandes problématiques actuelles au sein des fédérations sportives concerne la carrière et le taux d'abandon élevé chez les officiels de la plupart des pratiques sportives. Pour tenter de comprendre et d'expliquer ce qui incite à l'engagement ou à la résignation, la motivation à l'arbitrage est ainsi au cœur de 3 articles. Bjørn Tore Johansen examine d'abord les raisons de l'investissement d'arbitres de football élite et non-élite norvégiens avec une approche qualitative. Il extrait 3 principales causes - la passion, la motivation sociale et la santé - et discute les différences selon le niveau de pratique des arbitres. Ensuite, David Hancock, Donald J. Dawson et Denis Auger examinent les différentes motivations à débuter, à continuer et à arrêter l'arbitrage chez les officiels de 18 sports. À l'aide d'une méthode quantitative, ils soulignent l'importance de plusieurs variables clés telles que la motivation intrinsèque, les rôles déterminants du stress et du manque de respect. Enfin, à partir d'une revue de littérature et d'entretiens, Tom Parsons et Alan Bairner placent l'engagement d'arbitres de football anglais au cour de leur travail. L'ambition, la satisfaction et le support social sont discutés comme des éléments essentiels de l'investissement dans cette mission parfois difficile.

L'expertise et le stress des arbitres sont ensuite abordés. Alexandra Pizzera aborde d'abord l'expertise de l'officiel des pratiques sportives à travers le paradigme de la cognition incarnée, trop rarement convoqué lorsque les arbitres et leur processus décisionnels sont étudiés. Elle assume que la compréhension de l'expertise doit aussi passer par l'étude des expériences motrices, visuelles et d'arbitrage. Ensuite, Benoît Louvet, Mickaël Campo et Amaël André s'intéressent aux déterminants psychologiques dispositionnels et situationnels des stratégies de coping des arbitres de football. Après une analyse en équations structurales, les auteurs montrent que la perception de l'enjeu du match et l'intensité de l'anxiété prédisent les stratégies de coping tout en soulignant que le perfectionnisme reste la seule variable dispositionnelle influençant directement ces stratégies.

La communication est à la fois au cœur de problématiques de certaines fédérations sportives mais reste largement inexplorée par les chercheurs en sciences du sport. Trois articles abordent ainsi ce thème. Dans le premier, Ian Cunningham, Peter Simmons, Duncan Mascarenhas et Steve Redhead utilisent la sociologie dramaturgique de l'interaction sociale pour explorer les interactions entre les arbitres de sports collectifs et les joueurs. Ils observent que les joueurs tentent souvent d'influencer les arbitres par de multiples attitudes, améliorant ainsi la compréhension de la communication et des interactions sur le terrain. Simon Boyer, Géraldine Rix-Lièvre et Michel Recopé interrogent dans l'article suivant les ressorts et la coordination entre les membres des équipes arbitrales de football. Par l'intermédiaire d'entretiens d'auto-confrontation, les auteurs proposent une première cartographie de la contribution de chaque officiel à l'arbitrage d'une rencontre de football de haut-niveau. Finalement, François Borel-Hänni et Fabien Wille aborde la communication sous une autre forme. A partir d'archives et d'entretiens, il interroge les représentations médiatiques de l'arbitre de football et soulignent le rôle négatif - des médias télévisuels.

Ce numéro spécial se termine par une revue de littérature proposée par Matthew Weston sur les performances physiques et perceptivo-cognitives des arbitres de football en considérant ces deux aspects comme intimement liés. L'auteur aborde ainsi à la fois la quantification de la charge physique, le processus décisionnel, les programmes et les outils d'entraînement spécifiques. Il s'interroge finalement sur la fatigue, tout en soulignant les diverses difficultés à examiner son influence sur le jugement et la décision en situation, et les méthodes d'entraînement à la prise de décision.

Ce numéro spécial veut témoigner de la diversité et de la complémentarité des approches. L'arbitrage et l'officiel - arbitre ou juge - sont des objets de recherche pluridisciplinaire qui permettent d'aborder les décisions à long terme ou globales et celles à court terme ou locales. Les premières impliquent ainsi les choix de carrière, le désengagement et les transitions dans ce rôle à la fois spécifique et essentiel du domaine sportif. Les secondes concernent les décisions effectuées sur le terrain. Elles sont dynamiques, effectuées dans un environnement 
naturel alors que les contraintes temporelles, spatiales et d'incertitude sont multiples. Nous espérons que ce numéro thématique permettra de faire émerger de nouvelles problématiques de recherche dans le domaine des sciences du sport et du mouvement. Nous souhaiterions finalement remercier l'éditeur en chef de la revue Movement $\&$ Sport Sciences de nous avoir donner l'opportunité de coordonner ce numéro spécial. Nous espérons que ce dernier inspirera chercheurs et étudiants et favorisera les collaborations dans ce domaine.

Remerciements. Nous tenons à remercier Madame Emma Mosley, université de Bournemouth (UK) pour ses commentaires sur la première version de cette introduction.

Fabrice Dosseville ${ }^{1}$ et Sylvain Laborde ${ }^{2}$

1 Université de Caen Basse-Normandie

${ }^{2}$ German Sport University Cologne

\section{Bibliographie}

Alker, H.A., Straub, W.F., \& Leary, J. (1973). Achieving consistency: A Study of basketball officiating. Journal of Vocational Behavior, 3 (3), 335-343.

Bagnall, R.S., Brodersen, K., Champion, C.B., Erskine, A., \& Huebner, S.R. (2013). The Encyclopedia of Ancient History. Malden, MA: Wiley-Blackwell.

Bar-Eli, M., Plessner, H., \& Raab, M. (2010). Referees. In M. Bar-Eli, H. Plessner, \& M. Raab (Eds.), Judgment, decision-making and success in sport (pp. 123-144). Hoboken, NJ: John Wiley \& Sons, Ltd.

Brand, R., Schmidt, G., \& Schneeloch, Y. (2006). Sequential effects in elite basketball referees' foul decisions: An experimental study on the concept of game management. Journal of Sport and Exercise Psychology, 28 (1), 93-99.

Castagna, C., Abt, G., \& D'Ottavio, S. (2005). CompetitiveLevel Differences in Yo-Yo Intermittent Recovery and Twelve Minute Run Test Performance in Soccer Referees. Journal of Strength and Conditioning Research, 19 (4), 805-809.

Castagna, C., Abt, G., \& D’Ottavio, S. (2007). Physiological aspects of soccer refereeing performance and training. Sports Medicine, 37 (7), 625-647.

Catteeuw, P., Helsen, W., Gilis, B., \& Wagemans, J. (2009). Decision-making skills, role specificity, and deliberate practice in association football refereeing. Journal of Sports Sciences, 27 (11), 1125-1136.

Cunningham, I., Simmons, P., Mascarenhas, D. \& Redhead, S. (2014). Concepts of communication and player management in the development of sport officials. International Journal of Sport Communication, 7 (2), 166-187.

Dosseville, F., \& Garncarzyk, C. (2007). L'arbitrage des pratiques sportives : Jugement et décision. Bulletin de Psychologie, 60 (3), 225-237.
Dosseville, F., \& Laborde, S. (2011). Les facettes de l'arbitrage : Recherches et problématiques actuelles. Paris: Publibook, Collection Université: Sport \& Santé.

Dosseville, F., Laborde, S., \& Bernier, M. (2014). Athletes' expectations with regard to officiating competence. European Journal of Sport Science, 14 (S1), S448-S455.

Dosseville, F., Laborde, S., \& Garncarzyk, C. (2014). Current research in Sports officiating and decision-making. In C. Mohiyeddini (Ed.), Contemporary Topics and Trends in the Psychology of Sports (pp. 13-38). New York: Nova Publishers.

Dosseville, F., Laborde, S., \& Raab, M. (2011). Contextual and personal motor experience effects in judo referees' decisions. The Sport Psychologist, 25 (1), 67-81.

Dosseville, F., Laborde, S., Traclet, A., \& Edoh, K.P. (2011). Décisions arbitrales en football et performances physiques en situation écologique : Indicateurs comportementaux et physiologiques. STAPS, Revue Internationale des Sciences du Sport 83 de l'Education Physique, 93 (3), 51-60.

Findlay, L.C., \& Ste-Marie, D.M. (2004). A reputation bias in figure skating judging. Journal of Sport and Exercise Psychology, 26 (1), 154-166.

Fratzke, M.R. (1975). Personality and biographical traits of superior and average college basketball officials. The Research Quarterly, 46, 484-488.

Goumas, C. (2012). Home advantage and referee bias in European football. European Journal of Sport Science, 14 (sup1), S243-S249.

Gray, C.E., \& Wilson, P.M. (2008). The relationship between organizational commitment, perceived relatedness, and intentions to continue in Canadian track and field officials. Journal of Sport Behavior, 31, 44-63.

Grunska, J. (1999). Successful sports officiating. Champaign, IL: Human Kinetics.

Guillén, F., \& Feltz, D.L. (2011). A conceptual model of referee efficacy. Frontiers in Psychology, 2 (25), 1-5.

Helsen, W., \& Bultynck, J.-B. (2004). Physical and perceptual-cognitive demands of top-class refereeing in association football. Journal of Sports Sciences, 22 (2), 179-189.

Ittenbach, R.F., \& Eller, B.F. (1989). The Official Personality. Journal of Sport and Exercise Psychology, 11 (2), 119-119.

Jones, M.V., Paull, G.C., \& Erskine, J. (2002). The impact of a team's aggressive reputation on the decisions of association football referees. Journal of Sports Sciences, 20 (12), 991-1000.

Kaissidis, A., \& Anshel, M.H. (1993). Sources and intensity of acute stress in adolescent and adult Australian basketball referees: a preliminary study. Australian Journal of Science and Medicine in Sport, 25 (3), 97-103.

Krustrup, P., \& Bangsbo, J. (2001). Physiological demands of top-class soccer refereeing in relation to physical capacity: effect of intense intermittent exercise training. Journal of Sports Sciences, 19 (11), 881-891. 
Krustrup, P., Helsen, W., Randers, M.B., Christensen, J.F., MacDonald, C., Rebelo, A.N., \& Bangsbo, J. (2009). Activity profile and physical demands of football referees and assistant referees in international games. Journal of Sports Sciences, 27 (11), 1167-1176.

Larsen, J.D., \& Rainey, D.W. (1991). Judgment Bias in Baseball Umpires' First Base Calls: A Computer Simulation. Journal of Sport and Exercise Psychology, 13 (1), 75-79.

Leicht, A.S. (2008). Physiological demands of basketball refereeing during international competition. Journal of Science and Medicine in Sport, 11 (3), 357-360.

Louvet, B., Gaudreau, P., Menaut, A., Genty, J., \& Deneuve, P. (2009). Revisiting the changing and stable properties of coping utilization using latent class growth analysis: A longitudinal investigation with soccer referees. Psychology of Sport and Exercise, 10, 124-135.

MacMahon, C., \& Plessner, H. (2008). The sport officials in research and practice. In D. Farrow, J. Baker \& C. MacMahon (Eds.), Developing sport expertise. Researchers and coaches put theory into practice (pp. 172-192). Abingdon, Oxon: Routledge.

Martin, J., Smith, N.C., Tolfrey, K., \& Jones, A.M. (2001). Activity analysis of English premiership rugby football union refereeing. Ergonomics, 44 (12), 1069-1075.

Mascarenhas, D.R.D., Button, C., O'Hare, D., \& Dicks, M. (2009). Physical performance and decision making in association football referees: A naturalistic study. The Open Sports Sciences Journal, 2, 1-9.

Mascarenhas, D.R.D., Collins, D., \& Mortimer, P.W. (2005). Elite Refereeing Performance: Developing a model for sport science support. The Sport Psychologist, 19 (4), $364-379$.

Mellick, M.C., Fleming, S., \& Davies, G. (2007). An interpretive analysis of interpersonal communication: A case study from elite rugby union match officiating. International Journal of Performance Analysis in Sport, 7 (2), 92-105.

Nevill, A.M., Balmer, N.J., \& Williams, A.M. (1999). Crowd influence on decisions in association football. The Lancet, 353 (9162), 1416-1416.

Ollis, S., MacPherson, A., \& Collins, D. (2006). Expertise and talent development in rugby refereeing: an ethnographic enquiry. Journal of Sports Sciences, 24 (3), 309-322.

Oudejans, R.R.D., Verheijen, R., Bakker, F.C., Gerrits, J.C., Steinbrückner, M., \& Beek, P.J. (2000). Errors in judging 'offside' in football. Nature, 404, 33.

Philippe, F.L., Vallerand, R.J., Andrianarisoa, J., \& Brunel, P. (2009). Passion in referees: Examining their affective and cognitive experiences in sport situations. Journal of Sport and Exercise Psychology, 31 (1), 77-96.

Pizzera, A., \& Laborde, S. (2011). Comment devient-on expert en arbitrage? In F. Dosseville \& S. Laborde (Eds.), Les facettes de l'arbitrage: Recherches et problématiques actuelles (pp. 134-146). Paris : Publibook, Collection Université.

Pizzera, A., \& Raab, M. (2012). Perceptual judgments of sports officials are influenced by their motor and visual experience. Journal of Applied Sport Psychology, 24, 59-772.

Plessner, H., \& Haar, T. (2006). Sports performance judgments from a social cognitive perspective. Psychology of Sport and Exercise, 7, 555-575.

Simmons, P., \& Cunningham, I. (2013). Communication and sports officials. In P.M. Pedersen, Routledge handbook of sport communication (pp. 461-470). London : Routledge.

Souchon, N., Cabagno, G., Rascle, O., Traclet, A., Dosseville, F., \& Maio, G.R. (2009). Referees' decision making about transgressions: The influence of player at the highest national level. Psychology of Women Quarterly, 33 (4), 445-452.

Souchon, N. (2011). Stéréotypes, attitudes, préjugés et décisions arbitrales. In F. Dosseville \& S. Laborde (Eds.), Les facettes de l'arbitrage: Recherches et problématiques actuelles (pp. 113-113). Paris: Publibook, Collection Université.

Ste-Marie, D.M. (2003). Expertise in sport judges and referees. Circumventing information-processing limitations. In J.L. Starkes \& K.A. Ericsson (Eds.), Expert performance in sports: Advances in research on sport expertise (pp. 169-190). Champaign, IL: Human Kinetics.

Ste-Marie, D.M., \& Lee, T.D. (1991). Prior processing effects on gymnastic judging. Journal of Experimental Psychology: Learning, Memory \&6 Cognition, 17 (1), $126-136$.

Taylor, A.H., Daniel, J.V., Leith, L., \& Burke, R.J. (1990). Perceived stress, psychological burnout and paths to turnover intentions among sport officials. Journal of Applied Sport Psychology, 2 (1), 84-97.

Unkelbach, C., \& Memmert, D. (2010). Crowd noise as a cue in referee decisions contributes to the home advantage. Journal of Sport and Exercise Psychology, 32 (4), 483-498.

Voight, M. (2009). Sources of stress and coping strategies of US soccer officials. Stress and Health, 25 (1), 91-101.

Weinberg, R.S., \& Richardson, P.A. (1990). Psychology of officiating. Champaign, ILL: Leisure Press.

Weston, M., Castagna, C., Impellizzeri, F.M., Rampinini, E., \& Breivik, S. (2010). Ageing and physical match performance in English Premier League soccer referees. Journal of Science and Medicine in Sport, 13 (1), 96-100.

Weston, M., Drust, B., \& Gregson, W. (2011). Intensities of exercise during match-play in FA Premier League referees and players. Journal of Sports Sciences, 29 (5), 527-532.

Weston, M., Helsen, W., MacMahon, C., \& Kirkendall, D. (2004). The impact of specific high-intensity training sessions on football referees' fitness levels. American Journal of Sports Medicine, 32 (1 Suppl.), 54S-61S. 